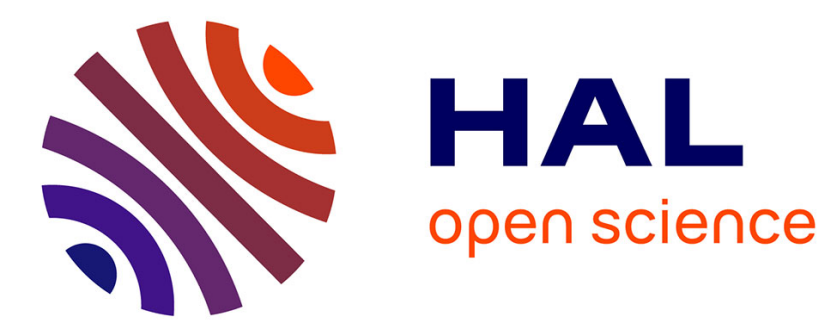

\title{
Common mathematical framework for stochastic reverberation models
}

\author{
Roland Badeau
}

\section{To cite this version:}

Roland Badeau. Common mathematical framework for stochastic reverberation models. Journal of the Acoustical Society of America, 2019, 145 (4), pp.2733-2745. 10.1121/1.5096153 . hal-01958485

\section{HAL Id: hal-01958485 \\ https://hal.science/hal-01958485}

Submitted on 9 May 2019

HAL is a multi-disciplinary open access archive for the deposit and dissemination of scientific research documents, whether they are published or not. The documents may come from teaching and research institutions in France or abroad, or from public or private research centers.
L'archive ouverte pluridisciplinaire HAL, est destinée au dépôt et à la diffusion de documents scientifiques de niveau recherche, publiés ou non, émanant des établissements d'enseignement et de recherche français ou étrangers, des laboratoires publics ou privés. 


\title{
Common mathematical framework for stochastic reverberation models
}

\author{
Roland Badeau ${ }^{1}$ \\ LTCI, Télécom ParisTech, Université Paris-Saclay, \\ Image, Data, Signal department (IDS), \\ 46 rue Barrault, 75013 Paris, France ${ }^{\text {a) }}$
}

In the field of room acoustics, it is well known that reverberation can be characterized statistically in a particular region of the time-frequency domain (after the transition time and above Schroeder's frequency). Since the 1950s, various formulas have been established, focusing on particular aspects of reverberation: exponential decay over time, correlations between frequencies, correlations between sensors at each frequency, and time-frequency distribution. In this paper, we introduce a stochastic reverberation model, that permits us to retrieve all these well-known results within a common mathematical framework. To the best of our knowledge, this is the first time that such a unification work is presented. The benefits are multiple: several formulas generalizing the classical results are established, that jointly characterize the spatial, temporal and spectral properties of late reverberation.

Keywords: Reverberation; Diffusion; Room impulse response; Stochastic models

a)roland.badeau@telecom-paristech.fr; This article appeared in The Journal of the Acoustical Society of America (Vol.145, No.4) and may be found at https://doi.org/10.1121/1.5096153. 
JASA/Common mathematical framework for stochastic reverberation models

\section{INTRODUCTION}

When a microphone records a sound produced by an audio source in a room, the received signal is made of several contributions (Kuttruff, 2014): firstly, the direct sound, that corresponds to the direct propagation of the sound wave from the source to the microphone, then a few early reflections, that are due to the sound wave reflections on the various room surfaces (walls, floor, ceiling...), and finally the late reverberation: after a time called transition time (Polack, 1992, 1993), reflections are so frequent that they form a continuum and, because the sound is partially absorbed by the room surfaces at every reflection, the sound level decays exponentially over time. This phenomenon is called reverberation, and it can be modeled as the convolution between the source signal and a causal room impulse response (RIR), made of a few isolated impulses before the transition time, and of a continuous, exponentially decaying, random process in late reverberation. The Fourier transform of the RIR is called room frequency response (RFR), and the modal theory (Balian and Bloch, 1970; Maa, 1939; Polack, 2007) shows that its profile is qualitatively similar to that of the RIR: below a frequency called Schroeder's frequency, the RFR is made of a few isolated modes, and above this frequency the modes become so dense that they can be represented as a continuous random process (Schroeder, 1962, 1987; Schroeder and Kuttruff, 1962).

To sum up, reverberation can be modeled as a stochastic process in a rectangular region of the time-frequency domain (Jot et al., 1997), as depicted in Fig. 1. If in addition the source and the microphones are located at least a half-wavelength away from the walls, 
then in this time-frequency region, the sound field can generally be approximated as diffuse (Cremer et al., 1982; Joyce, 1975; Schultz, 1971). Diffusion is a consequence of the reflections on the room surfaces not being specular (i.e. mirror-like), but rather scattered in various directions, as represented in Fig. 2. After many reflections, the sound field can be considered as isotropic: the sound waves come uniformly from all directions.

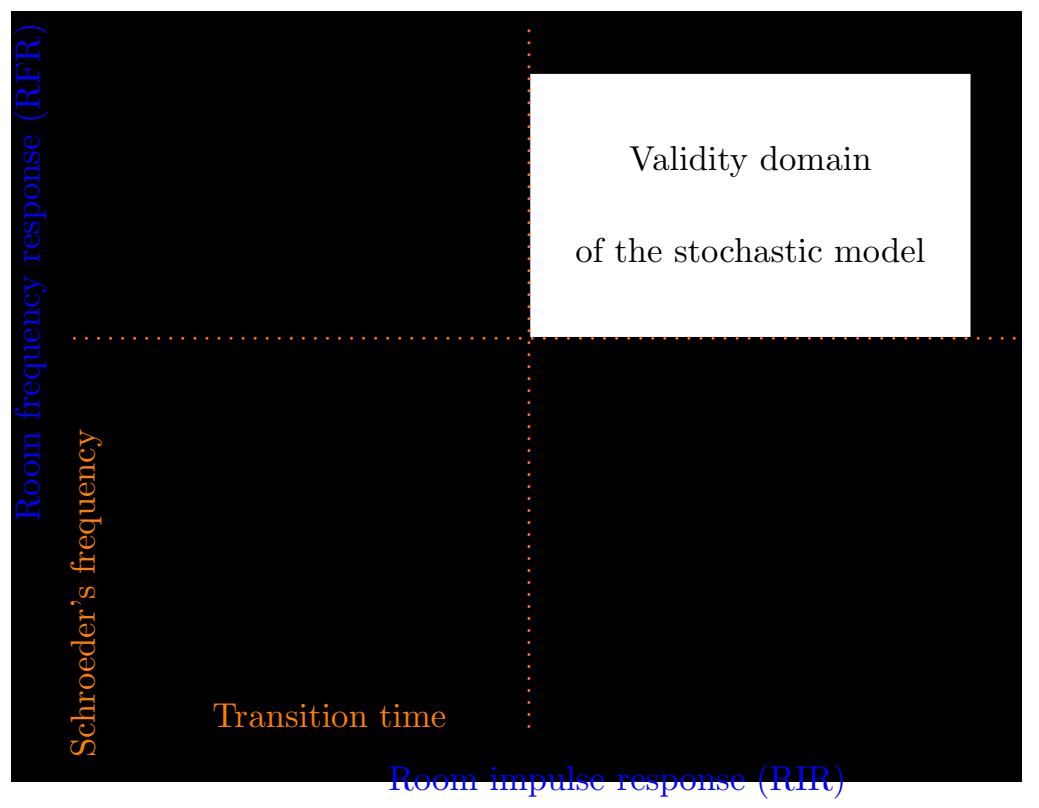

FIG. 1. Time-frequency profile of reverberation (adapted from (Jot et al., 1997, p. 30) and (Baskind, 2003, p. 20)).

Historically, the first stochastic reverberation model is due to Schroeder (1962) and Moorer (1979): the RIR at microphone $i$ is

$$
h_{i}(t)=b_{i}(t) e^{-\alpha t} \mathbf{1}_{t \geq 0}
$$

where $\alpha>0$ and $b_{i}(t)$ is a centered white Gaussian process. Parameter $\alpha$ is related to the reverberation time $T_{r}$ in seconds by the equation $T_{r}=\frac{3 \ln (10)}{\alpha}$. The Gaussian distribution of 
JASA/Common mathematical framework for stochastic reverberation models

42

43

44

$b_{i}(t)$ arises from the central limit theorem: in late reverberation, $h_{i}(t)$ is the sum of many independent contributions.

Schroeder (1962, 1987); Schroeder and Kuttruff (1962) also noticed that the independency of the samples $h_{i}(t)$ implies that the RFR, defined as their Fourier transform ${ }^{1} \mathcal{F}_{h_{i}}(f)$, is a stationary random process. From (1), he derived several formulas that can be summarized by expressing the complex autocorrelation function ${ }^{2}$ of $\mathcal{F}_{h_{i}}(f)$ :

$$
\operatorname{corr}\left[\mathcal{F}_{h_{i}}\left(f_{1}\right), \mathcal{F}_{h_{i}}\left(f_{2}\right)\right]=\frac{1}{1+\imath \pi \frac{f_{1}-f_{2}}{\alpha}}
$$

Following a similar approach in the spectral domain, under the diffuse field assumption, Cook et al. (1955) computed the correlation at frequency $f$ between two sensors at distance $D$ (with $c>0$ the speed of sound in the air):

$$
\operatorname{corr}\left[\mathcal{F}_{h_{1}}(f), \mathcal{F}_{h_{2}}(f)\right]=\operatorname{sinc}\left(\frac{2 \pi f D}{c}\right)
$$

Equation (3) was later generalized to combinations of pressure and velocity sensors (Jacobsen and Roisin, 2000) and to differential microphones (Elko, 2001).

Finally, Polack (1988) generalized model (1) by assuming that $b_{i}(t)$ is a centered stationary Gaussian process, whose power spectral density (PSD) $B(f)$ has slow variations ${ }^{3}$. Then he showed that the Wigner distribution ${ }^{4}$ (Cohen, 1989) of the RIR is

$$
\mathcal{W}_{h_{i}, h_{i}}(t, f)=B(f) e^{-2 \alpha t} \mathbf{1}_{t \geq 0}
$$

In order to account for the fact that the attenuation coefficient $\alpha$ actually depends on the frequency $f$, he also proposed an empirical generalization of (4):

$$
\mathcal{W}_{h_{i}, h_{i}}(t, f)=B(f) e^{-2 \alpha(f) t} \mathbf{1}_{t \geq 0}
$$


In other respects, based on the billiard theory, Polack (1992 1993) also showed that the durations of the various trajectories in a room, from a given source position to the microphone, are distributed according to a Poisson process (Chiu et al., 2013).

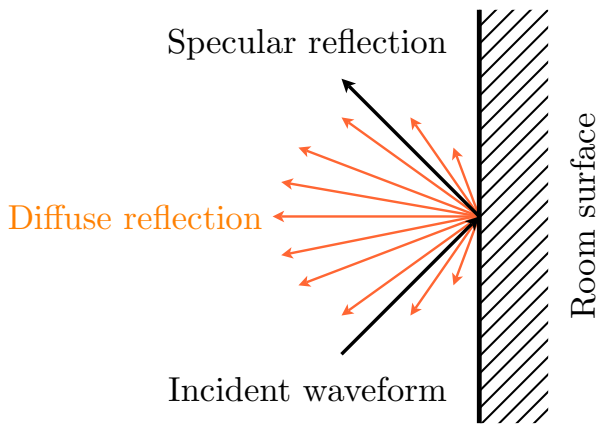

FIG. 2. Specular vs. diffuse reflection.

In this paper, we propose a unified stochastic model of reverberation, that will permit us to retrieve all formulas (1) to (4) in a common mathematical framework ${ }^{5}$, to establish a link with the Poisson distribution proposed by Polack, and to show how the probability distribution of the RIR, which is impulsive in early reverberation, converges to the Gaussian distribution in late reverberation. In addition, this model will also permit us to go deeper into the description of the statistical properties of the RIR over the space, time and frequency domains, and to prove several new results.

This paper is structured as follows: Section II presents important mathematical definitions and notation that will be used throughout the paper. Then our general stochastic reverberation model is introduced in Section III. The statistical properties of this model at one sensor are investigated in Section IV. The statistical relationships between two sensors are then analyzed in Section V. The new model is validated experimentally in Section VI, 
by comparing the predicted second order statistics with their estimates from a database of synthetic RIRs. Finally, some conclusions and perspectives are presented in Section VII. In order to make the main discussion as clear as possible, all mathematical proofs were moved to Appendices A to G.

\section{MATHEMATICAL DEFINITIONS}

- $\mathbb{N}$ : set of whole numbers

- $\mathbb{R}, \mathbb{C}$ : sets of real and complex numbers, respectively

- $\mathbb{R}_{+}$: set of nonnegative real numbers

- $\imath=\sqrt{-1}$ : imaginary unit

- $\boldsymbol{x}$ (bold font), $z$ (regular): vector and scalar, respectively

- $[a, b]$ : closed interval, including $a$ and $b \in \mathbb{R}$

- $] a, b[$ : open interval, excluding $a$ and $b \in \mathbb{R}$

- $L^{p}(V)$, where $V$ is a Borel set and $p \in \mathbb{N} \backslash\{0\}$ : Lebesgue space of measurable functions $f$ of support $V$, such that $\|f\|_{p}=\left(\int_{V}|f(\boldsymbol{x})|^{p} d \boldsymbol{x}\right)^{\frac{1}{p}}<+\infty$

- $L^{\infty}(V)$, where $V$ is a Borel set: Lebesgue space of essentially bounded functions $f$ of support $V$ (i.e. such that $\|f\|_{\infty}=\operatorname{ess}_{\sup }|f|<+\infty$ )

- $\delta$ : Dirac delta function

- $\|.\|_{2}$ : Euclidean/Hermitian norm of a vector or a function 
JASA/Common mathematical framework for stochastic reverberation models

- $\bar{z}$ : complex conjugate of $z \in \mathbb{C}$

- $\boldsymbol{x}^{\top}$ : transpose of vector $\boldsymbol{x}$

- $\mathcal{S}^{2}$ : unit sphere in $\mathbb{R}^{3}\left(\mathcal{S}^{2}=\left\{\boldsymbol{x} \in \mathbb{R}^{3} ;\|\boldsymbol{x}\|_{2}=1\right\}\right)$

- $\mathbb{E}[X]$ : expected value of a random variable $X$

- $\phi_{X}(\theta)=\mathbb{E}\left[e^{\imath \theta X}\right]$ : characteristic function of a real random variable $X$

- Covariance of two complex random variables $X$ and $Y$ :

$$
\operatorname{cov}[X, Y]=\mathbb{E}[(X-\mathbb{E}[X]) \overline{(Y-\mathbb{E}[Y])}]
$$

- $\operatorname{var}[X]=\operatorname{cov}[X, X]$ : variance of a random variable $X$

- Correlation of two complex random variables $X$ and $Y$ :

$$
\operatorname{corr}[X, Y]=\frac{\operatorname{cov}[X, Y]}{\sqrt{\operatorname{var}[X] \operatorname{var}[Y]}}
$$

- $\mathcal{P}(\lambda)$ : Poisson distribution of parameter $\lambda>0$ :

$$
N \sim \mathcal{P}(\lambda) \Leftrightarrow P(N=n)=e^{-\lambda} \frac{\lambda^{n}}{n !} \Leftrightarrow \phi_{N}(\theta)=e^{\lambda\left(e^{\imath \theta}-1\right)}
$$

- $\operatorname{sinc}(x)=\frac{\sin (x)}{x}$ : cardinal sine function

- $\mathbf{1}_{A}$ : indicator function of a set $A\left(\mathbf{1}_{A}(x)\right.$ is 1 if $x \in A$ or 0 if $\left.x \notin A\right)$

- $\widetilde{\psi}(t)=\overline{\psi(-t)}$ : conjugate and time-reverse of $\psi: \mathbb{R} \rightarrow \mathbb{C}$

- Convolution of two functions $\psi_{1}$ and $\psi_{2}: \mathbb{R} \rightarrow \mathbb{C}$ :

$$
\left(\psi_{1} * \psi_{2}\right)(t)=\int_{u \in \mathbb{R}} \psi_{1}(u) \psi_{2}(t-u) d u
$$


- Fourier transform of a function $\psi: \mathbb{R} \rightarrow \mathbb{C}$ :

$$
\mathcal{F}_{\psi}(f)=\int_{t \in \mathbb{R}} \psi(t) e^{-2 \imath \pi f t} d t \quad(f \in \mathbb{R})
$$

- Two-sided Laplace transform of a function $\psi: \mathbb{R} \rightarrow \mathbb{C}$ :

$$
\mathcal{L}_{\psi}(s)=\int_{t \in \mathbb{R}} \psi(t) e^{-s t} d t \quad(s \in \mathbb{C})
$$

- Wigner distribution (a.k.a. Wigner-Ville distribution) of two second-order random processes $\psi_{1}(t)$ and $\psi_{2}(t)$ :

$$
\mathcal{W}_{\psi_{1}, \psi_{2}}(t, f)=\int_{\mathbb{R}} \operatorname{cov}\left[\psi_{1}\left(t+\frac{u}{2}\right), \psi_{2}\left(t-\frac{u}{2}\right)\right] e^{-2 \imath \pi f u} d u
$$

\section{DEFINITION OF THE STOCHASTIC MODEL}

The model that we present in this section is based on the source image principle (Allen and Berkley, 1979; Kuttruff, 2014). As illustrated in Fig. 3 in the case of specular reflections in a rectangular room ${ }^{6}$, the trajectory inside the room from the real source to the microphone is equivalent to a virtual straight trajectory from a so-called source image which is outside the room. A remarkable property of this principle is that, regardless of the room dimensions, the density of the source images is uniform in the whole space: the number of source images contained in a given disk, of radius sufficiently larger than the room dimensions, is approximately invariant under any translation of this disk.

Since we aim to define a general stochastic reverberation model that holds for any room geometry, based on the billiard theory (Polack, 1992, 1993), we will consider that the positions of the source images are random and uniformly distributed (note that this assumption 
JASA/Common mathematical framework for stochastic reverberation models

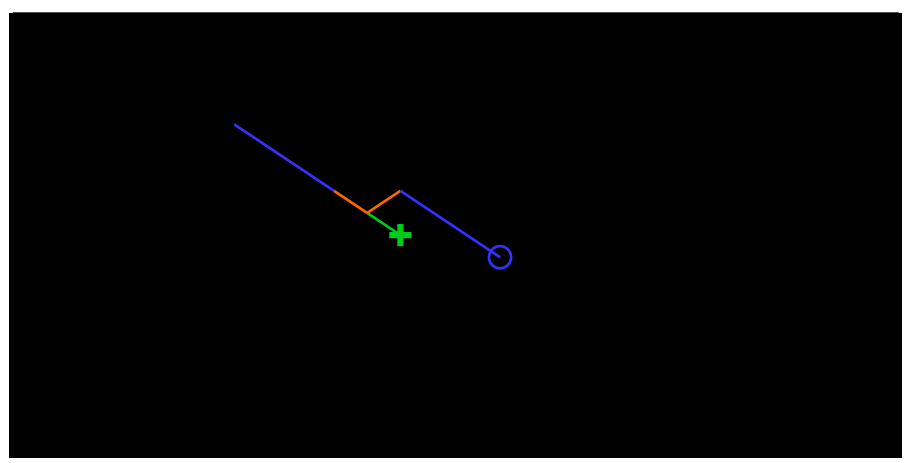

FIG. 3. Positions of microphone (plus sign), source (thick point) and source images (other points).

The original room walls are drawn with thick lines. A virtual straight trajectory from one source image to the microphone is drawn, along with the real trajectory in the original room.

is a fortiori valid in the case of a diffuse sound field, which is uniform). More precisely, given

a Borel set $V \subset \mathbb{R}^{3}$ of finite volume $|V|$, we will assume that the number $N(V)$ of source images contained in $V$ follows a Poisson distribution of parameter $\lambda|V|: N(V) \sim \mathcal{P}(\lambda|V|)$.

Mathematically, this is formalized through the concept of Poisson random measures with independent increments: given a non-negative, locally integrable function $\Lambda(\boldsymbol{x})$ on $\mathbb{R}^{p}$, the Poisson random increment $d N(\boldsymbol{x}) \sim \mathcal{P}(\Lambda(\boldsymbol{x}) d \boldsymbol{x})$ corresponds to an infinitesimal volume $|V|=d \boldsymbol{x}$. Then for any Borel set $V \subset \mathbb{R}^{p}$ of finite Lebesgue measure, the number $N(V)=\int_{V} d N(\boldsymbol{x})$ of points contained in $V$ follows a Poisson distribution of parameter $\int_{V} \Lambda(\boldsymbol{x}) d \boldsymbol{x}: \quad N(V) \sim \mathcal{P}\left(\int_{V} \Lambda(\boldsymbol{x}) d \boldsymbol{x}\right)$. In the proposed stochastic reverberation model, we will consider a spatially uniform distribution of the source images in the 3D-space, so that $p=3$ and $\Lambda(\boldsymbol{x})=\lambda>0$ is constant.

In other respects, we will assume that the sound waves undergo an exponential attenuation along their trajectories, that is due to the multiple reflections on the room surfaces 
and to the propagation in the air. In this paper we focus on the case of omnidirectional microphones, so we will further assume that this attenuation is isotropic (in accordance with the diffuse field approximation) and independent of the frequency. It will thus only depend on the length of the trajectory, as in (Polack, 1988).

Finally, we suppose that several microphones ${ }^{7}$ indexed by an integer $i$ are placed at arbitrary positions $\boldsymbol{x}_{i}$ in the room.

We end up with the following model:

$$
h_{i}(t)=\int_{\boldsymbol{x} \in \mathbb{R}^{3}} h_{i}(t, \boldsymbol{x}) e^{-\frac{\alpha}{c}\left\|\boldsymbol{x}-\boldsymbol{x}_{i}\right\|_{2}} d N(\boldsymbol{x}),
$$

where $h_{i}(t)$ is the RIR at microphone $i, \alpha>0$ is the attenuation coefficient (in $\mathrm{Hz}$ ), and $c>0$ is the speed of sound in the air (approximately $340 \mathrm{~m} / \mathrm{s}$ in usual conditions). The impulse $h_{i}(t, \boldsymbol{x})$, propagated from the source image at position $\boldsymbol{x}$, is modeled as a coherent sum of monochromatic spherical waves:

$$
h_{i}(t, \boldsymbol{x})=\int_{f \in \mathbb{R}} A(f) \frac{e^{2 \imath \pi f\left(t-\frac{\left\|\boldsymbol{x}-\boldsymbol{x}_{i}\right\|_{2}}{c}\right)}}{\left\|\boldsymbol{x}-\boldsymbol{x}_{i}\right\|_{2}} d f,
$$

where $A(f)$ is a linear-phase frequency response (in order to ensure coherence). In Appendix A, we show that (12) and (13) are equivalent to the following model:

Definition 1 (Unified stochastic reverberation model).

Let $\lambda>0, T>0, \alpha>0$, and $c>0$. Let $d N(\boldsymbol{x})$ be a uniform Poisson random measure on $\mathbb{R}^{3}$ with independent increments:

$$
d N(\boldsymbol{x}) \sim \mathcal{P}(\lambda d \boldsymbol{x})
$$


JASA/Common mathematical framework for stochastic reverberation models

149

Let $g(t) \in L^{\infty}([-T, T])$, such that

$$
\begin{array}{r}
\mathcal{F}_{g}(0)=\frac{d \mathcal{F}_{g}}{d f}(0)=0, \\
\forall f \in \mathbb{R}, \quad \mathcal{L}_{g}(\alpha+2 \imath \pi f) \geq 0 .
\end{array}
$$

At any sensor position $\boldsymbol{x}_{i} \in \mathbb{R}^{3}, h_{i}(t)$ is defined as

$$
\forall t \in \mathbb{R}, h_{i}(t)=e^{-\alpha(t-T)} b_{i}(t)
$$

where

$$
b_{i}(t)=\int_{\boldsymbol{x} \in \mathbb{R}^{3}} g\left(t-T-\frac{\left\|\boldsymbol{x}-\boldsymbol{x}_{i}\right\|_{2}}{c}\right) \frac{d N(\boldsymbol{x})}{\left\|\boldsymbol{x}-\boldsymbol{x}_{i}\right\|_{2}} .
$$

Equivalently, the Fourier transform of $h_{i}(t)$ is

$$
\mathcal{F}_{h_{i}}(f)=\mathcal{L}_{g}(\alpha+2 \imath \pi f) e^{-2 \imath \pi f T} \int_{\mathbb{R}^{3}} \frac{e^{-\frac{\alpha+2 \imath \pi f}{c}\left\|\boldsymbol{x}_{-} \boldsymbol{x}_{i}\right\|_{2}}}{\left\|\boldsymbol{x}-\boldsymbol{x}_{i}\right\|_{2}} d N(\boldsymbol{x})
$$

This definition calls for comments. Firstly, the linear-phase frequency response $A(f)$ in (13) was parameterized as

$$
A(f)=\mathcal{L}_{g}(\alpha+2 \imath \pi f) e^{-2 \imath \pi f T} .
$$

This technical definition aims to simplify the mathematical developments in the next sections. Secondly, any function $g \in L^{\infty}([-T, T])$ is such that $\mathcal{F}_{g}(f)$ and $f \mapsto \mathcal{L}_{g}(\alpha+2 \imath \pi f)$ are smooth, so $\mathcal{F}_{g}(0), \frac{d \mathcal{F}_{g}}{d f}(0)$ and $\mathcal{L}_{g}(\alpha+2 \imath \pi f)$ are well-defined. The constraints (15) and (16) imposed to $g$ are required to prove Lemma 1 and Proposition 2 in Section $I^{8}{ }^{8}$. Moreover, the support of $g$ is chosen so that $h_{i}(t)$ in $(17)$ and $b_{i}(t)$ in (18) are causal. Thirdly, the existence of functions $g$ that satisfy these constraints is guaranteed by Lemma 3 in Appendix A. 
JASA/Common mathematical framework for stochastic reverberation models

Now it is time to investigate the properties of this model. In Section IV, we will focus on one sensor at spatial position $\boldsymbol{x}_{i}$. Then in Section V, we will analyze the spatial relationships between two sensors at different positions $\boldsymbol{x}_{i}$ and $\boldsymbol{x}_{j}$.

\section{STATISTICAL PROPERTIES AT ONE SENSOR}

Let us first introduce an equivalent model definition:

Proposition 1 (Equivalent model definition at one sensor). With the same notation as in Definition 1, we have:

$$
\begin{aligned}
b_{i}(t) & =\int_{r \in \mathbb{R}_{+}} g\left(t-T-\frac{r}{c}\right) \frac{d N(r)}{r} \\
\mathcal{F}_{h_{i}}(f) & =\mathcal{L}_{g}(\alpha+2 \imath \pi f) e^{-2 \imath \pi f T} \int_{r \in \mathbb{R}_{+}} \frac{e^{-\frac{\alpha+2 \imath \pi f}{c} r} d N(r)}{r}
\end{aligned}
$$

where $d N(r)$ are independent Poisson increments on $\mathbb{R}_{+}$:

$$
d N(r) \sim \mathcal{P}\left(4 \pi \lambda r^{2} d r\right)
$$

Proposition 1 is proved in Appendix A. Let us now investigate the statistical properties of this model:

Lemma 1. The random process $b_{i}(t)$ introduced in Definition 1 is such that $\forall t \geq 2 T$,

1. $b_{i}(t)$ is a centered wide sense stationary (WSS) process $^{9}$, of autocovariance function

$$
\forall \tau \in \mathbb{R}, \Gamma(\tau)=\operatorname{cov}\left[b_{i}(t+\tau), b_{i}(t)\right]=4 \pi \lambda c(\widetilde{g} * g)(\tau)
$$

autocorrelation function

$$
\forall \tau \in \mathbb{R}, \gamma(\tau)=\operatorname{corr}\left[b_{i}(t+\tau), b_{i}(t)\right]=\frac{(\widetilde{g} * g)(\tau)}{\|g\|_{2}^{2}}
$$


JASA/Common mathematical framework for stochastic reverberation models

and power spectral density

$$
\forall f \in \mathbb{R}, B(f)=\mathcal{F}_{\Gamma}(f)=4 \pi \lambda c\left|\mathcal{F}_{g}(f)\right|^{2}
$$

2. When $t \rightarrow+\infty$ (i.e. $t \gg T)$, $b_{i}(t)$ converges in law to a stationary Gaussian process.

Lemma 1 is proved in Appendix C.

Proposition 2 (Statistical properties at one sensor position). With the notation introduced in Lemma 1, the reverberation model in Definition 1 has the following properties:

1. First order moments:

- in the spectral domain:

$$
\forall f \in \mathbb{R}, \mathbb{E}\left[\mathcal{F}_{h_{i}}(f)\right]=\frac{4 \pi \lambda c^{2} \mathcal{L}_{g}(\alpha+2 \imath \pi f) e^{-2 \imath \pi f T}}{(\alpha+2 \imath \pi f)^{2}}
$$

- in the time domain:

$$
\forall t \geq 2 T, \mathbb{E}\left[h_{i}(t)\right]=0
$$

2. Second order moments:

- in the spectral domain:

$$
\begin{aligned}
\forall f \in \mathbb{R}, \operatorname{var}\left[\mathcal{F}_{h_{i}}(f)\right] & =2 \pi \lambda c \mathcal{L}_{g}(\alpha+2 \imath \pi f)^{2} / \alpha \\
\forall f_{1}, f_{2} \in \mathbb{R}, \operatorname{corr}\left[\mathcal{F}_{h_{i}}\left(f_{1}\right), \mathcal{F}_{h_{i}}\left(f_{2}\right]\right) & =\frac{e^{-2 \imath \pi\left(f_{1}-f_{2}\right) T}}{1+\imath \pi \frac{f_{1}-f_{2}}{\alpha}}
\end{aligned}
$$

- in time domain:

$$
\begin{aligned}
\forall t \geq 2 T, \operatorname{var}\left[h_{i}(t)\right] & =4 \pi \lambda c\|g\|_{2}^{2} e^{-2 \alpha(t-T)} \\
\forall t_{1}, t_{2} \geq 2 T, \operatorname{corr}\left[h_{i}\left(t_{1}\right), h_{i}\left(t_{2}\right)\right] & =\gamma\left(t_{1}-t_{2}\right)
\end{aligned}
$$


JASA/Common mathematical framework for stochastic reverberation models

$$
\forall f \in \mathbb{R}, \forall t \geq 2 T, \mathcal{W}_{h_{i}, h_{i}}(t, f)=B(f) e^{-2 \alpha(t-T)} .
$$

3. Asymptotic normality: when $t \rightarrow+\infty, h_{i}(t)$ converges in law to a Gaussian process.

Proposition 2 is proved in Appendix D. Note that (26) and (15) show that $B(f)$ is very flat at $f=0: B(0)=\frac{d B}{d f}(0)=\frac{d^{2} B}{d f^{2}}(0)=\frac{d^{3} B}{d f^{3}}(0)=0$. The asymptotic normality is related to the central limit theorem: when $r$ becomes large, the volume contained between the spheres of radius $r$ and $r+d r$ increases as $r^{2} d r$, and so does the number of source images included in this volume as shown in (23), which leads to the addition of an increasing number of independent and identically distributed (i.i.d.) random increments $d N(\boldsymbol{x})$.

This proposition permits us to retrieve most of the classical results listed in the introduction. Firstly, $h_{i}(t)$ is centered for $t \geq 2 T$ (the fact that it is not centered for $t \in[0,2 T]$ explains why the expected value of the frequency response $\mathbb{E}\left[\mathcal{F}_{h_{i}}(f)\right]$ in $(27)$ is not zero). Secondly, (17) corresponds to Schroeder and Moorer's model defined in (1) when $T \rightarrow 0$ (in this case the process $b_{i}(t)$ becomes white, and it is Gaussian when $t \gg T$ ), and in the general case it is equivalent to Polack's model (Polack, 1988, chap. 1$)\left(b_{i}(t)\right.$ is a centered stationary Gaussian process when $t \gg T$ ). When $T \rightarrow 0$, (30) reduces to Schroeder's formula (2), which was indeed established by assuming that $b_{i}(t)$ is white. Finally, (33) is equivalent to Polack's time-frequency model defined in (4). To the best of our knowledge, the other formulas in Proposition 2 are novel. 
JASA/Common mathematical framework for stochastic reverberation models

\section{STATISTICAL PROPERTIES BETWEEN TWO SENSORS}

Let us now focus on the relationships between two sensors:

Lemma 2. Let us consider the model in Definition 1 at two positions $\boldsymbol{x}_{i}$ and $\boldsymbol{x}_{j} \in \mathbb{R}^{3}$. Let us define the rectangular window

$$
\forall t \in \mathbb{R}, w(t)=\frac{c}{2 D} \mathbf{1}_{\left[-\frac{D}{c}, \frac{D}{c}\right]}(t)
$$

where $D=\left\|\boldsymbol{x}_{i}-\boldsymbol{x}_{j}\right\|_{2}$. Then, in addition to the properties listed in Lemma 1, the random process $\boldsymbol{b}(t)=\left[b_{i}(t), b_{j}(t)\right]^{\top}$ is also such that

- $\forall t \geq 2 T+\frac{D}{c}, \boldsymbol{b}(t)$ is a centered $W S S$ process $^{9}$, of cross-autocovariance function

$$
\forall \tau \in \mathbb{R}, \Gamma_{i, j}(\tau)=\operatorname{cov}\left[b_{i}(t+\tau), b_{j}(t)\right]=w * \Gamma(\tau)
$$

cross-autocorrelation function

$$
\forall \tau \in \mathbb{R}, \gamma_{i, j}(\tau)=\operatorname{corr}\left[b_{i}(t+\tau), b_{j}(t)\right]=w * \gamma(\tau)
$$

and cross-power spectral density

$$
\forall f \in \mathbb{R}, B_{i, j}(f)=\mathcal{F}_{\Gamma_{i, j}}(f)=B(f) \operatorname{sinc}\left(\frac{2 \pi f D}{c}\right) .
$$

- When $t \rightarrow+\infty$ (i.e. $t \gg T)$, b(t) converges in law to a stationary Gaussian process.

Lemma 2 is proved in Appendix F.

Proposition 3 (Statistical properties between two sensors).

Let us consider the reverberation model in Definition 1 at two positions $\boldsymbol{x}_{i}$ and $\boldsymbol{x}_{j} \in \mathbb{R}^{3}$, and the rectangular window $w(t)$ defined in (34) with $D=\left\|\boldsymbol{x}_{i}-\boldsymbol{x}_{j}\right\|_{2}$. Then, with the notation 
JASA/Common mathematical framework for stochastic reverberation models

217

introduced in Lemma 1 and in addition to the properties listed in Proposition 2, we also have:

- in the spectral domain:

$$
\forall f_{1}, f_{2} \in \mathbb{R}, \operatorname{corr}\left[\mathcal{F}_{h_{i}}\left(f_{1}\right), \mathcal{F}_{h_{j}}\left(f_{2}\right)\right]=\frac{e^{-\frac{\alpha D}{c}-2 \imath \pi\left(f_{1}-f_{2}\right)\left(T+\frac{D}{2 c}\right)} \operatorname{sinc}\left(\frac{\pi\left(f_{1}+f_{2}\right) D}{c}\right)}{1+\imath \pi \frac{f_{1}-f_{2}}{\alpha}}
$$

- in the time domain:

$$
\forall t_{1}, t_{2} \geq 2 T+\frac{D}{c}, \operatorname{corr}\left[h_{i}\left(t_{1}\right), h_{j}\left(t_{2}\right)\right]=w * \gamma\left(t_{1}-t_{2}\right)
$$

- in the time-frequency domain:

$$
\forall f \in \mathbb{R}, \forall t \geq 2 T+\frac{D}{2 c}, \mathcal{W}_{h_{i}, h_{j}}(t, f)=B(f) e^{-2 \alpha(t-T)} \operatorname{sinc}\left(\frac{2 \pi f D}{c}\right) .
$$

- Asymptotic normality: when $t \rightarrow+\infty, \boldsymbol{h}(t)$ converges in law to a Gaussian process.

Proposition 3 is proved in Appendix G. Applying equation (38) to $f_{1}=f_{2}=f$, we get Cook's formula (3) when $\alpha \rightarrow 0$ (no exponential decay). This formula was indeed originally proved by considering plane waves under a far-field assumption (Cook et al., 1955). Besides, equation (39) shows that $h_{i}(t)$ and $h_{j}(t)$ are correlated on a time interval that corresponds to the wave propagation from one sensor to the other. To the best of our knowledge, all formulas in Proposition 3 are novel.

\section{SIMULATION RESULTS}

In Propositions 2 and 3, we have listed several statistical properties of the reverberation model introduced in Definition 1. Some of these properties are already-known experimental 
facts, such as the zero mean and asymptotic Gaussianity of late reverberation. In this section, we aim to experimentally validate the second-order statistics specific to this new stochastic reverberation model, that were not predicted by the existing models listed in Section I. Firstly, we note that at the second order, the spectral domain, time domain, and time-frequency domain formulas in both Propositions 2 and 3 are mutually equivalent; we can thus focus on the time-frequency domain only. Secondly, from (33) and (40), we get the closed-form expression of the time-frequency correlation: $\forall f \in \mathbb{R}, \forall t \geq 2 T+\frac{D}{2 c}$,

$$
\frac{\mathcal{W}_{h_{i}, h_{j}}(t, f)}{\mathcal{W}_{h_{i}, h_{i}}(t, f)}=\operatorname{sinc}\left(\frac{2 \pi f D}{c}\right) \text {. }
$$

Since (33) was already known (Polack, 1988) and since (40) can actually be deduced from (33) and (41), the novelty regarding the second order statistics of late reverberation in Propositions 2 and 3 lies in the single equation (41). Therefore we just need to experimentally validate (41).

For this purpose, we need an experimental setup with the greatest spatial diversity possible: a high number (typically a thousand) of RIRs, with random source position $\boldsymbol{x}$, random middle position of the sensors $\frac{\boldsymbol{x}_{i}+\boldsymbol{x}_{j}}{2}$, random orientation of the sensor axis $\frac{\boldsymbol{x}_{j}-\boldsymbol{x}_{i}}{D}$, for a fixed distance $\left\|\boldsymbol{x}_{j}-\boldsymbol{x}_{i}\right\|_{2}=D$. Because we do not have access to a suitable database of measured RIRs, we used synthetic RIRs instead. These synthetic RIRs were generated with the Roomsimove toolbox (Vincent and Campbell, 2008), which is a state-of-the-art RIR generator based on the source image principle. Roomsimove is dedicated to parallelepipedic ("shoebox") rooms and applies high-pass filtering above $20 \mathrm{~Hz}$. We used it with the default physical parameters (humidity: $40 \%$, temperature: $20^{\circ} \mathrm{C}$, speed of sound: $c=343 \mathrm{~m} / \mathrm{s}$ ), to simulate a shoebox room matching the two properties of the classroom described in (Kut- 
truff, 2014, p. 84): a volume of $200 \mathrm{~m}^{3}$ (the room dimensions are $7.4 \mathrm{~m} \times 9 \mathrm{~m} \times 3 \mathrm{~m}$ ), and a reverberation time of $1 \mathrm{~s}$ (the absorption coefficient is set to 0.13 for all room surfaces). For this setup, Schroeder's frequency is about $140 \mathrm{~Hz}$. In accordance with our stochastic model, we considered omnidirectional sources and omnidirectional microphones. We thus generated $M=1000$ RIRs sampled at $16 \mathrm{kHz}$, with random source positions and random middle positions of the sensors (both uniformly distributed inside the room volume), random orientation of the sensor axis (uniformly distributed on the unit sphere), for a fixed microphone distance $D=20 \mathrm{~cm}$.

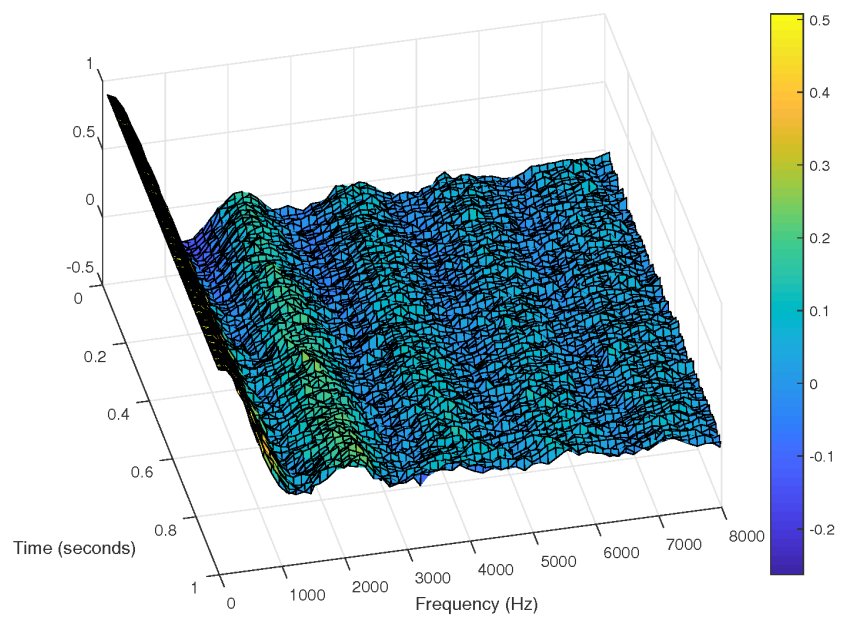

FIG. 4. Time-frequency correlation between sensors of the late RIR

Fig. 4 represents the estimate of the time-frequency correlation (41) that we obtained with this experimental setup. The Wigner distributions $\mathcal{W}_{h_{i}, h_{i}}(t, f)$ and $\mathcal{W}_{h_{i}, h_{j}}(t, f)$ were estimated as $\widehat{\mathcal{W}}_{h_{i}, h_{i}}(t, f)=\frac{1}{M} \sum_{m=1}^{M}\left|S_{h_{i}}^{(m)}(t, f)\right|^{2}$ and $\widehat{\mathcal{W}}_{h_{i}, h_{j}}(t, f)=\frac{1}{M} \sum_{m=1}^{M} S_{h_{i}}^{(m)}(t, f) \overline{S_{h_{j}}^{(m)}(t, f)}$, where $S_{h_{i}}^{(m)}(t, f)$ (resp. $\left.S_{h_{j}}^{(m)}(t, f)\right)$ is the short time Fourier transform (STFT) of the $m^{\text {th }}$ generated RIR $h_{i}^{(m)}(t)$ (resp. $h_{j}^{(m)}(t)$ ), computed with a 128-sample long Hann window and 
JASA/Common mathematical framework for stochastic reverberation models
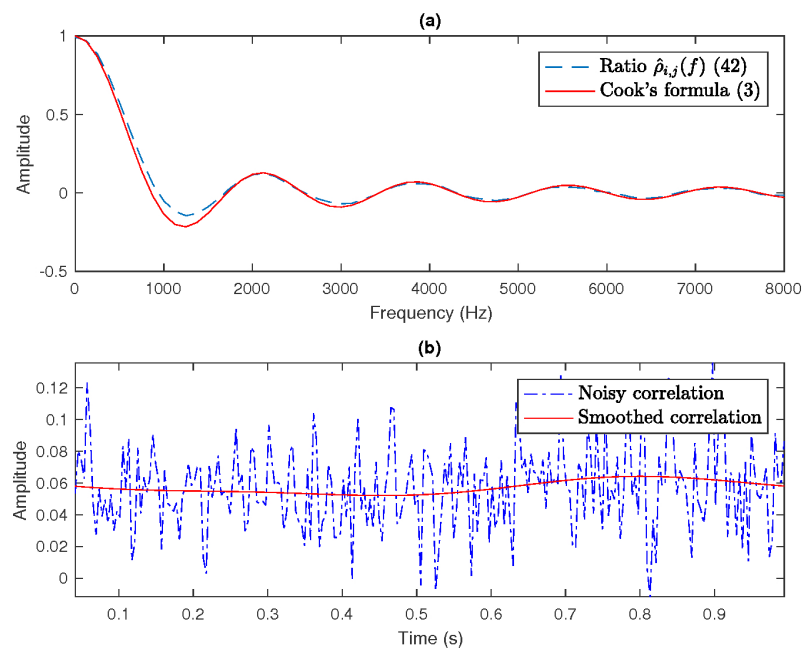

FIG. 5. Correlation between sensors of the late RFR over frequency (a) and over time (b)

an overlap of 64 samples in the time domain. The distributions $\widehat{\mathcal{W}}_{h_{i}, h_{i}}(t, f)$ and $\widehat{\mathcal{W}}_{h_{i}, h_{j}}(t, f)$ obtained in this way are smoothed estimates of $\mathcal{W}_{h_{i}, h_{i}}(t, f)$ and $\mathcal{W}_{h_{i}, h_{j}}(t, f)$ in the timefrequency domain (Cohen, 1989). We observe in Fig. 4 that the estimated time-frequency correlation is noisy but, as expected, it seems approximately constant as a function of time, and looks like a cardinal sine function as a function of frequency.

In order to accurately compare the frequency variation with Cook's formula (3), we computed the ratio $\hat{\rho}_{i, j}(f)$ of the projections of the estimated distributions onto the frequency axis, i.e.

$$
\hat{\rho}_{i, j}(f)=\frac{\int_{\mathbb{R}} \widehat{\mathcal{W}}_{h_{i}, h_{j}}(t, f) d t}{\int_{\mathbb{R}} \widehat{\mathcal{W}}_{h_{i}, h_{i}}(t, f) d t}
$$

(represented by the dashed curve in Fig. 5-(a)), which is an estimate of (3) (the cardinal sine function is represented by the solid curve in Fig. 5-(a)). We can observe a good match between the two curves ${ }^{10}$. Finally, the dash-dotted curve in Fig. 5-(b) represents, as a function of time, the same noisy estimate of the time-frequency correlation as in Fig. 4, at 
a fixed frequency $f=3860 \mathrm{~Hz}$ (which corresponds to a local maximum of the cardinal sine function in Fig. 5-(a)), and the solid curve represents a smoothed (i.e. low-pass filtered) version of this noisy signal. We can observe that the average time-frequency correlation is indeed approximately independent of time.

\section{CONCLUSION AND PERSPECTIVES}

In this paper, we proposed a new stochastic model of reverberation, that permitted us to retrieve various well-known results within a common framework. This unification work resulted in several new results, that jointly characterize the properties of late reverberation in the space, time, and frequency domains. The most noticeable result in our opinion is (40), which very simply makes the connection between Polack's time-frequency model (4) and Cook's formula (3).

Although this model was motivated by physical assumptions that only hold in a particular region of the time-frequency domain (after the transition time and above Schroeder's frequency), from a signal processing perspective however, one of its most interesting features is that it is also applicable before the transition time and below Schroeder's frequency. Indeed, since the parameter of the Poisson distribution $d N(r)$ in (23) increases quadratically with the distance $r$, the model permits us to describe both the impulsiveness of the RIR before the transition time, and its asymptotic normality in late reverberation. However, because it does not take the exact geometry of the acoustic scene into account (i.e. the room geometry and the source and sensors positions), it is able to capture neither the accurate temporal localization in the RIR of the isolated peaks related to early reflections before the 
JASA/Common mathematical framework for stochastic reverberation models

transition time, nor the spectral localization of the isolated room modes below Schroeder's

frequency. In practice, this blindness to such fine details does not prevent us to estimate the model parameters from the full RIRs, in the whole time-frequency domain. The missing geometrical information can still be recovered afterwards in an ad-hoc manner, for instance by estimating the Poisson random measure $d N(\boldsymbol{x})$ given the observed RIRs. Indeed, the knowledge of $d N(\boldsymbol{x})$ permits us to identify the spatial positions of the source images, which carry the geometrical information related to both the early reflections and the room modes. Finally, we end up with a stochastic model involving very few parameters $(\alpha, \lambda$, filter $g$, and the distances between microphones), that is able to describe reverberation in the whole time-frequency domain. We thus believe that this model has an interesting potential in a variety of signal processing applications.

However this reverberation model, as it is presented in this paper, is not yet suitable for modeling real RIRs. Indeed, one assumption has to be relaxed: the attenuation coefficient $\alpha$ is not constant but rather depends on frequency in practice, as in Polack's generalized time-frequency model (5). In a future paper, we will thus present a generalization of the proposed model where we will introduce a frequency-varying attenuation coefficient. second generalization of this model would be to represent acoustic fields that are not perfectly diffuse. Finally, the generalization to directional microphones is straightforward, by using the same approach as presented in (Elko, 2001).

Our future contributions will also focus on the signal processing aspects of this work: we will propose a fast algorithm for estimating the parameters of the model in discrete time. 
JASA/Common mathematical framework for stochastic reverberation models

Finally, our ultimate goal is to investigate the potential of this model in applications such as source separation, dereverberation, and synthetic reverberation.

\section{ACKNOWLEDGMENTS}

Ahead of this work, I had some very interesting and inspiring discussions about reverberation with several persons that I would like to thank here, including my former $\mathrm{PhD}$ students Simon Leglaive and Arthur Belhomme, and my colleagues Gaël Richard, Yves Grenier, Laurent Girin, Antoine Liutkus, Philippe Depalle and Xavier Boutillon.

\section{APPENDIX A: PROOFS FOR THE DEFINITION OF THE STOCHASTIC MODEL}

The following lemma aims to prove the existence of functions $g$ that satisfy conditions (15) and (16) in Definition 1.

Lemma 3. Let $\psi(t) \in L^{2}([0, T])$ with $T>0$, such that $\int_{t=0}^{T} \psi(t) e^{\alpha t} d t=\int_{t=0}^{T} \psi(t) e^{-\alpha t} d t=0$, where $\alpha \in \mathbb{R}^{11}$. Let $g(t)=(\widetilde{\psi} * \psi)(t) e^{\alpha(t-T)}$. Then function $g$ satisfies conditions (15) and (16) in Definition 1.

Proof of Lemma 3. Since $\psi(t) \in L^{2}([0, T])$, function $g$ is continuous and bounded. Firstly, $\mathcal{F}_{g}(f)=e^{-\alpha T} \overline{\mathcal{L}_{\psi}(\alpha+2 \imath \pi f)} \mathcal{L}_{\psi}(-\alpha+2 \imath \pi f)$. Moreover, since $\psi(t)$ has finite support, both functions $f \mapsto \mathcal{L}_{\psi}(-\alpha+2 \imath \pi f)$ and $f \mapsto \mathcal{L}_{\psi}(\alpha+2 \imath \pi f)$ are infinitely differentiable. In addition, $\mathcal{L}_{\psi}(-\alpha)=\mathcal{L}_{\psi}(\alpha)=0$, which finally proves (15). Secondly, $\mathcal{L}_{g}(\alpha+2 \imath \pi f)=e^{-\alpha T}\left|\mathcal{F}_{\psi}(f)\right|^{2}$, which proves (16). 
We can now derive equations (17), (18) and (19) in Definition 1. By substituting (13) and (20) into (12), we get

$$
h_{i}(t)=\int_{f \in \mathbb{R}} \mathcal{L}_{g}(\alpha+2 \imath \pi f) e^{2 \imath \pi f(t-T)} \int_{\boldsymbol{x} \in \mathbb{R}^{3}} \frac{e^{-\frac{\alpha+2 \imath \pi f}{c}\left\|\boldsymbol{x}-\boldsymbol{x}_{i}\right\|_{2}}}{\left\|\boldsymbol{x}-\boldsymbol{x}_{i}\right\|_{2}} d N(\boldsymbol{x}) d f
$$

which shows that $h_{i}(t)$ as defined in (12) is the inverse Fourier transform of (19) and therefore proves (19). Besides, applying the Fourier transform (9) to (17) also leads to (19), which proves the equivalence between (17)-(18), and (12)-(13)-(20).

Finally, let us prove Proposition 1.

Proof of Proposition 1. Let $d N(r)=\int_{\boldsymbol{u} \in \mathcal{S}^{2}} d N(r \boldsymbol{u})$. Then (14) implies (23). The change of variables $\boldsymbol{x}=\boldsymbol{x}_{i}+r \boldsymbol{u}$ with $r \in \mathbb{R}_{+}$and $\boldsymbol{u} \in \mathcal{S}^{2}$ in (18) and (19) leads to (21) and (22).

\section{APPENDIX B: PROPERTIES OF THE POISSON RANDOM MEASURE}

The following Proposition 4 characterizes the distribution of the Poisson random measure introduced in Section III, by providing the closed-form expression of the characteristic functional of this random measure:

Proposition 4. Let $d N(\boldsymbol{x})$ be a Poisson random measure on $\mathbb{R}^{p}$ with independent increments: $d N(\boldsymbol{x}) \sim \mathcal{P}(\Lambda(\boldsymbol{x}) d \boldsymbol{x})$, where $\Lambda$ is a non-negative, locally integrable function. We consider the stochastic integral $I=\int \psi(\boldsymbol{x}) d N(\boldsymbol{x})$, where $\psi$ is an essentially bounded realvalued function with compact support, and its characteristic function $\phi_{I}(\theta)=\mathbb{E}\left[e^{\imath \theta I}\right]$. Then we have

$$
\forall \theta \in \mathbb{R}, \phi_{I}(\theta)=\exp \left(\int\left(e^{\imath \theta \psi(\boldsymbol{x})}-1\right) \Lambda(\boldsymbol{x}) d \boldsymbol{x}\right)
$$


JASA/Common mathematical framework for stochastic reverberation models

353

354

which yields

$$
\forall \theta \in \mathbb{R}, \ln \phi_{I}(\theta)=\sum_{n=1}^{+\infty} \frac{\imath^{n}}{n !} \theta^{n} \kappa_{n}
$$

where $\forall n \in \mathbb{N} \backslash\{0\}$,

$$
\kappa_{n}=\int \psi(\boldsymbol{x})^{n} \Lambda(\boldsymbol{x}) d \boldsymbol{x}
$$

is the $n$-th order cumulant of I. In particular, we get

$$
\mathbb{E}[I]=\int \psi(\boldsymbol{x}) \Lambda(\boldsymbol{x}) d \boldsymbol{x} .
$$

Proof. In the particular case where $\psi$ is the indicator function of a Borel set $V$ of finite Lebesgue measure, we have $I=N(V) \sim \mathcal{P}\left(\int_{V} \Lambda(\boldsymbol{x}) d \boldsymbol{x}\right)$. Therefore (8) yields (B1), because both members of (B1) are equal to $\exp \left(\left(e^{\imath \theta}-1\right) \int_{V} \Lambda(\boldsymbol{x}) d \boldsymbol{x}\right)$. Consequently, since (B1) holds for any indicator function and since the increments $d N(\boldsymbol{x})$ are assumed independent, it is easy to show that (B1) also holds for simple functions ${ }^{12}$. Following the construction of the Lebesgue integral, we conclude that (B1) also holds for any essentially bounded function $\psi$ with compact support.

Then the Taylor series expansion of the second exponential function in (B1) yields $\forall \theta \in \mathbb{R}$,

$$
\ln \phi_{I}(\theta)=\sum_{n=1}^{+\infty} \frac{\imath^{n}}{n !} \theta^{n} \int \psi(\boldsymbol{x})^{n} \Lambda(\boldsymbol{x}) d \boldsymbol{x}
$$

which proves (B2). Note that the permutation of the integral and the sum in (B5) is allowed thanks to Fubini's theorem, because $\sum_{n=1}^{+\infty} \frac{1}{n !}|\theta|^{n} \int|\psi(\boldsymbol{x})|^{n} \Lambda(\boldsymbol{x}) d \boldsymbol{x} \leq e^{|\theta|\|\psi\|_{\infty}} \int_{V} \Lambda(\boldsymbol{x}) d \boldsymbol{x}<$ $+\infty$, since $\psi$ is essentially bounded, $V=\operatorname{support}(\psi)$ is compact, and $\Lambda$ is locally integrable. In particular, for $n=1$, we get $\mathbb{E}[I]=\kappa_{1}$ thus (B3) yields (B4). 
JASA/Common mathematical framework for stochastic reverberation models

The following Proposition 5 provides the closed-form expression of the joint cumulants of two stochastic integrals involving the Poisson random measure:

Proposition 5. Let $d N(\boldsymbol{x})$ be a Poisson random measure on $\mathbb{R}^{p}$ with independent increments: $d N(\boldsymbol{x}) \sim \mathcal{P}(\Lambda(\boldsymbol{x}) d \boldsymbol{x})$, where $\Lambda$ is a non-negative, locally integrable function. We consider two stochastic integrals $I_{1}=\int \psi_{1}(\boldsymbol{x}) d N(\boldsymbol{x})$ and $I_{2}=\int \psi_{2}(\boldsymbol{x}) d N(\boldsymbol{x})$, where $\psi_{1}$ and $\psi_{2}$ are essentially bounded real-valued functions with compact support, and their joint characteristic function $\phi_{I_{1}, I_{2}}\left(\theta_{1}, \theta_{2}\right)=\mathbb{E}\left[e^{\imath\left(\theta_{1} I_{1}+\theta_{2} I_{2}\right.}\right]$. Then we have

$$
\forall \theta_{1}, \theta_{2} \in \mathbb{R}, \ln \left(\phi_{I_{1}, I_{2}}\left(\theta_{1}, \theta_{2}\right)\right)=\sum_{n=1}^{+\infty} \frac{\imath^{n}}{n !} \sum_{k=0}^{n}\left(\begin{array}{l}
n \\
k
\end{array}\right) \theta_{1}^{k} \theta_{2}^{n-k} \kappa_{k, n-k},
$$

where $\forall n_{1}, n_{2} \in \mathbb{N}$ such that $n_{1}+n_{2} \geq 1$,

$$
\kappa_{n_{1}, n_{2}}=\int \psi_{1}(\boldsymbol{x})^{n_{1}} \psi_{2}(\boldsymbol{x})^{n_{2}} \Lambda(\boldsymbol{x}) d \boldsymbol{x}
$$

is the $\left(n_{1}, n_{2}\right)$-th order joint cumulant of $\left(I_{1}, I_{2}\right)$. In particular, we get

$$
\operatorname{cov}\left[I_{1}, I_{2}\right]=\int \psi_{1}(\boldsymbol{x}) \psi_{2}(\boldsymbol{x}) \Lambda(\boldsymbol{x}) d \boldsymbol{x}
$$

Proof. By applying (B1) to $\theta=1$ and to $\psi(\boldsymbol{x})=\theta_{1} \psi_{1}(\boldsymbol{x})+\theta_{2} \psi_{2}(\boldsymbol{x})$, we get $\forall \theta_{1}, \theta_{2} \in \mathbb{R}$,

$$
\begin{aligned}
\ln \phi_{I_{1}, I_{2}}\left(\theta_{1}, \theta_{2}\right) & =\int\left(e^{\imath\left(\theta_{1} \psi_{1}(\boldsymbol{x})+\theta_{2} \psi_{2}(\boldsymbol{x})\right)}-1\right) \Lambda(\boldsymbol{x}) d \boldsymbol{x} \\
& =\sum_{n=1}^{+\infty} \frac{\imath^{n}}{n !} \int\left(\theta_{1} \psi_{1}(\boldsymbol{x})+\theta_{2} \psi_{2}(\boldsymbol{x})\right)^{n} \Lambda(\boldsymbol{x}) d \boldsymbol{x} \\
& =\sum_{n=1}^{+\infty} \frac{\imath^{n}}{n !} \sum_{k=0}^{n}\left(\begin{array}{l}
n \\
k
\end{array}\right) \theta_{1}^{k} \theta_{2}^{n-k} \int \psi_{1}(\boldsymbol{x})^{k} \psi_{2}(\boldsymbol{x})^{n-k} \Lambda(\boldsymbol{x}) d \boldsymbol{x}
\end{aligned}
$$

which proves (B6). Note that the permutation of the integral and the two sums in (B9) is allowed thanks to Fubini's theorem, because

$\sum_{n=1}^{+\infty} \frac{1}{n !} \sum_{k=0}^{n}\left(\begin{array}{l}n \\ k\end{array}\right)\left|\theta_{1}\right|^{k}\left|\theta_{2}\right|^{n-k} \int\left|\psi_{1}(\boldsymbol{x})\right|^{k}\left|\psi_{2}(\boldsymbol{x})\right|^{n-k} \Lambda(\boldsymbol{x}) d \boldsymbol{x} \leq e^{\left|\theta_{1}\right|\left\|\psi_{1}\right\|_{\infty}+\left|\theta_{2}\right|\left\|\psi_{1}\right\|_{\infty}} \int_{V} \Lambda(\boldsymbol{x}) d \boldsymbol{x}<+\infty$ 
since $\psi_{1}$ and $\psi_{2}$ are essentially bounded, $V=\operatorname{support}\left(\psi_{1}\right) \cup \operatorname{support}\left(\psi_{2}\right)$ is compact, and $\Lambda$ is locally integrable. In particular, for $n_{1}=1$ and $n_{2}=1$, we get $\operatorname{cov}\left[I_{1}, I_{2}\right]=\kappa_{1,1}$, thus (B7) yields (B8).

\section{APPENDIX C: PROOF OF LEMMA 1}

\section{Wide sense stationarity}

\section{a. $\quad$ First order moments}

Considering the Poisson random measure $d N(r)$ defined in (23), equation (B4) in Proposition 4 , applied to $p=1$ and to function $\Lambda(r)=4 \pi \lambda r^{2}$ defined on $\mathbb{R}_{+}$, shows that for any essentially bounded (possibly complex) function $r \mapsto \psi(r)$ with compact support in $\mathbb{R}_{+}, \mathbb{E}\left[\int_{r \in \mathbb{R}_{+}} \psi(r) d N(r)\right]=4 \pi \lambda \int_{r \in \mathbb{R}_{+}} \psi(r) r^{2} d r$, which we will rewrite in the symbolic, more compact form:

$$
\mathbb{E}[d N(r)]=4 \pi \lambda r^{2} d r
$$

From (C1), since $\int_{u=-T}^{\min (t-T, T)}|g(u)|(t-T-u) d u<+\infty$ (with the change of variable $\left.r \mapsto u=t-T-\frac{r}{c}\right)$, Fubini's theorem shows that we can permute the mathematical expectation with the integral over $r$ in (21), leading to:

$$
\mathbb{E}\left[b_{i}(t)\right]=4 \pi \lambda c^{2}\left(\int_{u=-T}^{\min (t-T, T)}(t-T-u) g(u) d u\right)
$$

Finally, substituting (15) into (C2) shows that $\forall t \geq 2 T, \mathbb{E}\left[b_{i}(t)\right]=0$. 
JASA/Common mathematical framework for stochastic reverberation models

where $\kappa_{n}(t)$ is the $n$-th order cumulant of $b_{i}(t)$, whose expression is given by (B3):

$$
\kappa_{n}(t)=4 \pi \lambda \int_{r \in \mathbb{R}_{+}} g\left(t-T-\frac{r}{c}\right)^{n} r^{2-n} d r
$$

The change of variables $r=c(t-T-u)$ in (C5) implies

$$
\forall t \geq 2 T, \kappa_{n}(t)=4 \pi \lambda c^{3-n} \int_{u=-T}^{T} g(u)^{n}(t-T-u)^{2-n} d u
$$

From (C3), since $\int_{u \in \mathbb{R}}|g(u+\tau) g(u)| d u<+\infty$ (with the change of variable $r \mapsto u=$ $\left.t-T-\frac{r}{c}\right)$, Fubini's theorem shows that we can permute the mathematical expectation with the integral over $r$ in (21), leading to (24), which jointly proves both (25) and (26).

\section{Asymptotic normality}

From $(21), b_{i}(t)$ can be written as the stochastic integral $I$ in Proposition 4 , applied to $p=1$, to function $\Lambda(r)=4 \pi \lambda r^{2}$ defined on $\mathbb{R}_{+}$, and to $\psi(r)=\frac{g\left(t-T-\frac{r}{c}\right)}{r}$. Then (B2) yields

$$
\forall \theta \in \mathbb{R}, \ln \left(\phi_{b_{i}(t)}(\theta)\right)=\sum_{n=1}^{+\infty} \frac{(\imath \theta)^{n}}{n !} \kappa_{n}(t)
$$


Therefore, $\forall n \geq 2, \forall t>2 T$,

$$
\left|\kappa_{n}(t)\right| \leq \frac{4 \pi \lambda c^{3-n} \int_{u=-T}^{T}|g(u)|^{n} d u}{(t-2 T)^{n-2}}
$$

Let $\varepsilon>0$. Substituting (C6) into (C4), we get: $\forall t \geq(2+\varepsilon) T$,

$$
\left|\ln \left(\phi_{b_{i}(t)}(\theta)\right)-\left(\imath \theta \kappa_{1}(t)-\frac{\theta^{2}}{2} \kappa_{2}(t)\right)\right| \leq \frac{\psi(\theta)}{t-2 T} \underset{t \rightarrow+\infty}{\longrightarrow} 0,
$$

where $\psi(\theta)=4 \pi \lambda(\varepsilon c T)^{3} \int_{u=-T}^{T}\left(e^{\left|\frac{\theta g(u)}{\varepsilon c T}\right|}-\sum_{n=0}^{2} \frac{\left|\frac{\theta g(u)}{\varepsilon c T}\right|^{n}}{n !}\right) d u$.

409

Therefore the characteristic function of $b_{i}(t)$ converges pointwise to that of the normal distribution when $t \rightarrow+\infty$, which proves that $b_{i}(t)$ is asymptotically normally distributed. In the same way, it can be proved ${ }^{13}$ that the random variables $b_{i}\left(t+t_{1}\right) \ldots b_{i}\left(t+t_{K}\right)$ for all $K \in \mathbb{N}$ and $t, t_{1} \ldots t_{K} \in \mathbb{R}$ are jointly normally distributed when $t \rightarrow+\infty$ and $t_{1} \ldots t_{K}$ are fixed, which shows that $b_{i}(t)$ converges in law to a stationary Gaussian process.

\section{APPENDIX D: PROOF OF PROPOSITION 2}

\section{First order moments}

From (C1), since $\int_{r \in \mathbb{R}_{+}} e^{-\frac{\alpha}{c} r} r d r<+\infty$, Fubini's theorem shows that we can permute the mathematical expectation with the integral over $r$ in (22), leading to (27). Besides, since $\mathbb{E}\left[b_{i}(t)\right]=0$ as shown in Lemma 1, equation (17) implies (28). 
JASA/Common mathematical framework for stochastic reverberation models

where

$$
I(t, f)=\int_{u \in \mathbb{R}} g\left(t+\frac{u}{2}\right) g\left(t-\frac{u}{2}\right) e^{-2 \imath \pi f u} d u=\mathcal{W}_{g, g}(t, f) .
$$

\section{c. In the time-frequency domain}

Finally, from (C3) and (17), since

$$
\int_{u \in \mathbb{R}} \int_{r \in \mathbb{R}_{+}}\left|g\left(t+\frac{u}{2}-T-\frac{r}{c}\right) g\left(t-\frac{u}{2}-T-\frac{r}{c}\right)\right| d r d u<+\infty
$$

Fubini's theorem shows that we can permute the mathematical expectation with the integrals over $r$ in (21) and over $u$ in (11) (with $\psi_{1}=\psi_{2}=h_{i}$ ), leading to:

$$
\mathcal{W}_{h_{i}, h_{i}}(t, f)=4 \pi \lambda e^{-2 \alpha(t-T)} \int_{r \in \mathbb{R}_{+}} I\left(t-T-\frac{r}{c}, f\right) d r
$$


In order to conclude, we will use two properties of the Wigner distribution. Firstly, the time support of $\mathcal{W}_{g, g}$ is the same as that of $g$. Secondly, the projection property shows that $\int_{t \in \mathbb{R}} \mathcal{W}_{g, g}(t, f) d t=\left|\mathcal{F}_{g}(f)\right|^{2}$. Therefore substituting (D3) and (26) into (D2) finally implies (33).

\section{Asymptotic normality}

Since $b_{i}(t)$ converges in law to a stationary Gaussian process as shown in Lemma 1, equation (17) shows that $h_{i}(t)$ converges in law to a Gaussian process.

\section{APPENDIX E: GEOMETRY WITH TWO MICROPHONES}

Let $\boldsymbol{x}_{i}, \boldsymbol{x}_{j} \in \mathbb{R}^{3}$. Let $\xi_{1}, \xi_{2}: \mathbb{R}_{+} \rightarrow \mathbb{C}$. In the next sections, we will have to compute several integrals of the form:

$$
J_{\xi_{1}, \xi_{2}}=\int_{\boldsymbol{x} \in \mathbb{R}^{3}} \frac{\xi_{1}\left(\left\|\boldsymbol{x}-\boldsymbol{x}_{i}\right\|_{2}\right)}{\left\|\boldsymbol{x}-\boldsymbol{x}_{i}\right\|_{2}} \frac{\overline{\xi_{2}\left(\left\|\boldsymbol{x}-\boldsymbol{x}_{j}\right\|_{2}\right)}}{\left\|\boldsymbol{x}-\boldsymbol{x}_{j}\right\|_{2}} d \boldsymbol{x} \text {. }
$$

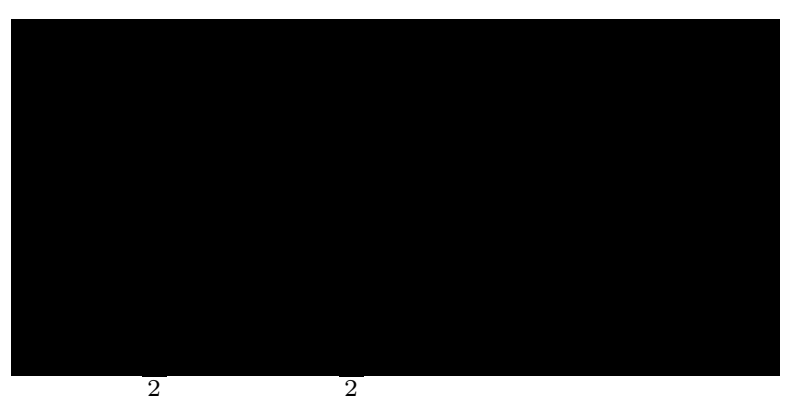

FIG. 6. Geometry with two microphones at $\boldsymbol{x}_{i}, \boldsymbol{x}_{j}$ and a source image at $\boldsymbol{x}$.

To compute such an integral, we will use the spherical coordinates $(r, \theta, \varphi)$, as illustrated in Fig. 6, where $\theta=0$ corresponds to the direction of vector $\boldsymbol{x}_{j}-\boldsymbol{x}_{i}$, and the origin of the 
coordinates is the middle of the line segment $\left[\boldsymbol{x}_{i}, \boldsymbol{x}_{j}\right]$ (so that $\frac{\boldsymbol{x}_{i}+\boldsymbol{x}_{j}}{2}=0$ as represented in

Fig. 6). We thus get $\boldsymbol{x}=[r \sin (\theta) \cos (\varphi), r \sin (\theta) \sin (\varphi), r \cos (\theta)]^{\top}$ and $d \boldsymbol{x}=r^{2} d r \sin (\theta) d \theta d \varphi$, with $r \in \mathbb{R}_{+}, \theta \in[0, \pi]$ and $\varphi \in[0,2 \pi]$. Moreover, as can be seen in Fig. 6, we have

- $\left\|\boldsymbol{x}-\boldsymbol{x}_{i}\right\|_{2}=\sqrt{r^{2}+\frac{D^{2}}{4}+r D \cos (\theta)}$,

- $\left\|\boldsymbol{x}-\boldsymbol{x}_{j}\right\|_{2}=\sqrt{r^{2}+\frac{D^{2}}{4}-r D \cos (\theta)}$.

By substitution into (E1), we get

$$
J_{\xi_{1}, \xi_{2}}=2 \pi \int_{r=0}^{+\infty} \int_{\theta=0}^{\pi} \frac{\xi_{1}\left(\sqrt{r^{2}+\frac{D^{2}}{4}+r D \cos (\theta)}\right)}{\sqrt{r^{2}+\frac{D^{2}}{4}+r D \cos (\theta)}} \frac{\overline{\xi_{2}\left(\sqrt{r^{2}+\frac{D^{2}}{4}-r D \cos (\theta)}\right)}}{\sqrt{r^{2}+\frac{D^{2}}{4}-r D \cos (\theta)}} r^{2} d r \sin (\theta) d \theta .
$$

Finally, we make a last change of variables, that is also illustrated in Fig. 6:

$$
\begin{aligned}
& \rho=\frac{\left\|\boldsymbol{x}-\boldsymbol{x}_{i}\right\|_{2}+\left\|\boldsymbol{x}-\boldsymbol{x}_{j}\right\|_{2}}{2}=\frac{\sqrt{r^{2}+\frac{D^{2}}{4}+r D \cos (\theta)}+\sqrt{r^{2}+\frac{D^{2}}{4}-r D \cos (\theta)}}{2}, \\
& v=\left\|\boldsymbol{x}-\boldsymbol{x}_{i}\right\|_{2}-\left\|\boldsymbol{x}-\boldsymbol{x}_{j}\right\|_{2}=\sqrt{r^{2}+\frac{D^{2}}{4}+r D \cos (\theta)}-\sqrt{r^{2}+\frac{D^{2}}{4}-r D \cos (\theta)},
\end{aligned}
$$

which is such that $\rho \in\left[\frac{D}{2},+\infty[, v \in[-D, D]\right.$, and

$$
\frac{r^{2} d r \sin (\theta) d \theta}{\sqrt{r^{2}+\frac{D^{2}}{4}+r D \cos (\theta)} \sqrt{r^{2}+\frac{D^{2}}{4}-r D \cos (\theta)}}=\frac{d \rho d v}{D} .
$$

Equation (E2) thus becomes

$$
J_{\xi_{1}, \xi_{2}}=\frac{2 \pi}{D} \int_{\rho=\frac{D}{2}}^{+\infty} \int_{v=-D}^{D} \xi_{1}\left(\rho+\frac{v}{2}\right) \overline{\xi_{2}\left(\rho-\frac{v}{2}\right)} d \rho d v
$$

\section{APPENDIX F: PROOF OF LEMMA 2}

\section{Wide sense stationarity}

Considering the Poisson random measure $d N(\boldsymbol{x})$ defined in (14), equation (B8) in Proposition 5, applied to $p=3$ and to the constant function $\Lambda(\boldsymbol{x})=\lambda$, shows that for any 
JASA/Common mathematical framework for stochastic reverberation models

453

454

essentially bounded (possibly complex) functions $\boldsymbol{x} \mapsto \psi_{1}(\boldsymbol{x})$ and $\boldsymbol{x} \mapsto \psi_{2}(\boldsymbol{x})$ with compact supports in $\mathbb{R}^{3}, \operatorname{cov}\left[\int_{\boldsymbol{x}_{1} \in \mathbb{R}^{3}} \psi_{1}\left(\boldsymbol{x}_{1}\right) d N\left(\boldsymbol{x}_{1}\right), \int_{\boldsymbol{x}_{2} \in \mathbb{R}^{3}} \psi_{2}\left(\boldsymbol{x}_{2}\right) d N\left(\boldsymbol{x}_{2}\right)\right]=\lambda \int_{\boldsymbol{x} \in \mathbb{R}^{3}} \psi_{1}(\boldsymbol{x}) \overline{\psi_{2}(\boldsymbol{x})} d \boldsymbol{x}$, which we will rewrite in the symbolic, more compact form:

$$
\operatorname{cov}\left[d N\left(\boldsymbol{x}_{1}\right), d N\left(\boldsymbol{x}_{2}\right)\right]=\lambda \delta\left(\boldsymbol{x}_{2}-\boldsymbol{x}_{1}\right) d \boldsymbol{x}_{1} d \boldsymbol{x}_{2} .
$$

From (F1), since

$$
\int_{\boldsymbol{x} \in \mathbb{R}^{3}} \frac{\left|g\left(t_{1}-T-\frac{\left\|\boldsymbol{x}-\boldsymbol{x}_{i}\right\|_{2}}{c}\right) g\left(t_{2}-T-\frac{\left\|\boldsymbol{x}-\boldsymbol{x}_{j}\right\|_{2}}{c}\right)\right|}{\left\|\boldsymbol{x}-\boldsymbol{x}_{i}\right\|_{2}\left\|\boldsymbol{x}-\boldsymbol{x}_{j}\right\|_{2}} d \boldsymbol{x}<+\infty
$$

Fubini's theorem shows that we can permute the mathematical expectation with the integral over $\boldsymbol{x}$ in (18), leading to:

$$
\operatorname{cov}\left[b_{i}\left(t_{1}\right), b_{j}\left(t_{2}\right)\right]=\lambda J_{\xi_{1}, \xi_{2}}
$$

where $\xi_{1}(r)=g\left(t_{1}-T-\frac{r}{c}\right), \xi_{2}(r)=g\left(t_{2}-T-\frac{r}{c}\right)$, and $J_{\xi_{1}, \xi_{2}}$ was defined in (E1). Then substituting (E3) into (F2) shows that

$$
\operatorname{cov}\left[b_{i}\left(t_{1}\right), b_{j}\left(t_{2}\right)\right]=4 \pi \lambda \int_{\rho=\frac{D}{2}}^{+\infty} I\left(t_{1}-T-\frac{\rho}{c}, t_{2}-T-\frac{\rho}{c}\right) d \rho
$$

where

$$
I\left(t_{1}, t_{2}\right)=\frac{1}{2 D} \int_{v=-D}^{D} g\left(t_{1}-\frac{v}{2 c}\right) g\left(t_{2}+\frac{v}{2 c}\right) d v
$$

With $v=c u$, substituting (34) into (F4) proves that

$$
I\left(t_{1}, t_{2}\right)=\int_{u \in \mathbb{R}} g\left(t_{1}-\frac{u}{2}\right) g\left(t_{2}+\frac{u}{2}\right) w(u) d u .
$$

Substituting (F5) into (F3) proves (35), which with (25) implies (36). Moreover, applying the Fourier transform (9) to (35) and substituting (26) leads to (37). 
JASA/Common mathematical framework for stochastic reverberation models

\section{Asymptotic normality}

Let $\boldsymbol{b}(t)=\left[b_{i}(t), b_{j}(t)\right]^{\top}$. From $(18), b_{i}(t)$ (resp. $\left.b_{j}(t)\right)$ can be written as the stochastic integral $I_{1}$ (resp. $I_{2}$ ) in Proposition 5 , applied to $p=3$, to the constant function $\Lambda(\boldsymbol{x})=\lambda$, and to $\psi_{1}(\boldsymbol{x})=\frac{g\left(t-T-\frac{\left\|\boldsymbol{x}-\boldsymbol{x}_{i}\right\|_{2}}{c}\right)}{\left\|\boldsymbol{x}-\boldsymbol{x}_{i}\right\|_{2}}$ and $\psi_{2}(\boldsymbol{x})=\frac{g\left(t-T-\frac{\left\|\boldsymbol{x}-\boldsymbol{x}_{j}\right\|_{2}}{c}\right)}{\left\|\boldsymbol{x}-\boldsymbol{x}_{j}\right\|_{2}}$. Then (B6) yields

$$
\forall \theta_{1}, \theta_{2} \in \mathbb{R}, \ln \left(\phi_{\boldsymbol{b}(t)}\left(\theta_{1}, \theta_{2}\right)\right)=\sum_{n=1}^{+\infty} \frac{\imath^{n}}{n !} \sum_{k=0}^{n}\left(\begin{array}{l}
n \\
k
\end{array}\right) \theta_{1}^{k} \theta_{2}^{n-k} \kappa_{k, n-k}(t)
$$

where $\kappa_{n_{1}, n_{2}}(t)$ is the $\left(n_{1}, n_{2}\right)$-th order cumulant of $\boldsymbol{b}(t)$, whose expression is given by $(\mathrm{B} 7)$ :

$$
\kappa_{n_{1}, n_{2}}(t)=\lambda J_{\xi_{1}, \xi_{2}}
$$

where $\xi_{1}(r)=\frac{\left(g\left(t-T-\frac{r}{c}\right)\right)^{n_{1}}}{r^{n_{1}-1}}, \xi_{2}(r)=\frac{\left(g\left(t-T-\frac{r}{c}\right)\right)^{n_{2}}}{r^{n_{2}-1}}$, and $J_{\xi_{1}, \xi_{2}}$ was defined in (E1).

Then substituting (E3) into (F7) shows that

$$
\kappa_{n_{1}, n_{2}}(t)=\frac{2 \pi \lambda}{D} \int_{\rho=\frac{D}{2}}^{+\infty} \int_{v=-D}^{D} \frac{\left(g\left(t-T-\frac{\rho+\frac{v}{2}}{c}\right)\right)^{n_{1}}}{\left(\rho+\frac{v}{2}\right)^{n_{1}-1}} \frac{\left.\left(t-T-\frac{\rho-\frac{v}{2}}{c}\right)\right)^{n_{2}}}{\left(\rho-\frac{v}{2}\right)^{n_{2}-1}} d \rho d v .
$$

The change of variables $\rho=c(t-T-u)$ and $v=c w$ in (F8) implies that $\forall t \geq 2 T+\frac{D}{c}$,

$$
\kappa_{n_{1}, n_{2}}(t)=\frac{2 \pi \lambda c^{4-n_{1}-n_{2}}}{D} \int_{u=-T-\frac{D}{2 c}}^{T+\frac{D}{2 c}} \int_{w=-\frac{D}{c}}^{\frac{D}{c}} \frac{\left(g\left(u-\frac{w}{2}\right)\right)^{n_{1}}}{\left(t-T-u+\frac{w}{2}\right)^{n_{1}-1}} \frac{\left(g\left(u+\frac{w}{2}\right)\right)^{n_{2}}}{\left(t-T-u-\frac{w}{2}\right)^{n_{2}-1}} d u d w .
$$

Therefore $\forall n_{1}+n_{2} \geq 2, \forall t \geq 2 T+\frac{D}{c}$,

$$
\left|\kappa_{n_{1}, n_{2}}(t)\right| \leq \frac{2 \pi \lambda c^{4-n_{1}-n_{2}}}{D} \frac{\int_{u=-T-\frac{D}{2 c}}^{T+\frac{D}{2 c}} \int_{w=-\frac{D}{c}}^{\frac{D}{c}}\left|g\left(u-\frac{w}{2}\right)\right|^{n_{1}}\left|g\left(u+\frac{w}{2}\right)\right|^{n_{2}} d u d w}{(t-2 T)^{n_{1}+n_{2}-2}}
$$

Substituting (F9) into (F6), we get $\forall t \geq 2 T+\frac{D}{c}$,

$$
\left|\ln \left(\phi_{\boldsymbol{b}(t)}(\theta)\right)-\sum_{n=1}^{2} \frac{\imath^{n}}{n !} \sum_{k=0}^{n}\left(\begin{array}{l}
n \\
k
\end{array}\right) \theta_{1}^{k} \theta_{2}^{n-k} \kappa_{k, n-k}(t)\right| \leq \frac{1}{(t-2 T)} \psi\left(\theta_{1}, \theta_{2}\right) \underset{t \rightarrow+\infty}{\longrightarrow} 0,
$$


where $\psi\left(\theta_{1}, \theta_{2}\right)=2 \pi \lambda c D^{2} \int_{u=-T-\frac{D}{2 c}}^{T+\frac{D}{2 c}} \int_{w=-\frac{D}{c}}^{\frac{D}{c}} e^{\frac{\left|\theta_{1} g\left(u-\frac{w}{2}\right)\right|+\left|\theta_{2} g\left(u+\frac{w}{2}\right)\right|}{D}}-\sum_{n=0}^{2} \frac{1}{n !}\left(\frac{\left|\theta_{1} g\left(u-\frac{w}{2}\right)\right|+\left|\theta_{2} g\left(u+\frac{w}{2}\right)\right|}{D}\right)^{n} d u d w$.

Therefore the characteristic function of $\boldsymbol{b}(t)$ converges pointwise to that of the normal distribution when $t \rightarrow+\infty$, which proves that $\boldsymbol{b}(t)$ is asymptotically normally distributed. In the same way, it can be proved ${ }^{13}$ that the random variables $\boldsymbol{b}\left(t+t_{1}\right) \ldots \boldsymbol{b}\left(t+t_{K}\right)$ for all $K \in \mathbb{N}$ and $t, t_{1} \ldots t_{K} \in \mathbb{R}$ are jointly normally distributed when $t \rightarrow+\infty$ and $t_{1} \ldots t_{K}$ are fixed, which proves that $\boldsymbol{b}(t)$ converges in law to a stationary Gaussian process.

\section{APPENDIX G: PROOF OF PROPOSITION 3}

\section{In the spectral domain}

From (F1), since

$$
\int_{\mathbb{R}^{3}} \frac{e^{-\frac{\alpha}{c}\left\|\boldsymbol{x}-\boldsymbol{x}_{i}\right\|_{2}}}{\left\|\boldsymbol{x}-\boldsymbol{x}_{i}\right\|_{2}} \frac{e^{-\frac{\alpha}{c}\left\|\boldsymbol{x}-\boldsymbol{x}_{j}\right\|_{2}}}{\left\|\boldsymbol{x}-\boldsymbol{x}_{j}\right\|_{2}} d \boldsymbol{x}<+\infty
$$

Fubini's theorem shows that we can permute the mathematical expectation with the integral over $\boldsymbol{x}$ in (19), leading to:

$$
\operatorname{cov}\left[\mathcal{F}_{h_{i}}\left(f_{1}\right), \mathcal{F}_{h_{j}}\left(f_{2}\right)\right]=\lambda \mathcal{L}_{g}\left(\alpha+2 \imath \pi f_{1}\right) \mathcal{L}_{g}\left(\alpha+2 \imath \pi f_{2}\right) e^{-2 \imath \pi\left(f_{1}-f_{2}\right) T} J_{\xi_{1}, \xi_{2}},
$$

where $\xi_{1}(r)=e^{-\frac{\alpha+22 \pi f_{1}}{c} r}, \xi_{2}(r)=e^{-\frac{\alpha+22 \pi f_{2}}{c} r}$, and $J_{\xi_{1}, \xi_{2}}$ was defined in (E1). Then equation (E3) shows that

$$
J_{\xi_{1}, \xi_{2}}=\frac{2 \pi}{D} \int_{\rho=\frac{D}{2}}^{+\infty} e^{-2 \frac{\alpha+\imath \pi\left(f_{1}-f_{2}\right)}{c} \rho} d \rho \int_{v=-D}^{D} e^{-2 \imath \pi \frac{f_{1}+f_{2}}{2 c} v} d v=2 \pi c \frac{e^{-\frac{\alpha+\imath \pi\left(f_{1}-f_{2}\right)}{c} D}}{\alpha+\imath \pi\left(f_{1}-f_{2}\right)} \operatorname{sinc}\left(2 \pi \frac{f_{1}+f_{2}}{2 c} D\right) .
$$

By substituting (G2) and (29) into (G1), we finally get (38). 
JASA/Common mathematical framework for stochastic reverberation models

496

\section{In the time domain}

Equations (17) and (35) prove that

$$
\operatorname{cov}\left[h_{i}\left(t_{1}\right), h_{j}\left(t_{2}\right)\right]=4 \pi \lambda c e^{-2 \alpha\left(\frac{t_{1}+t_{2}}{2}-T\right)} w * \widetilde{g} * g\left(t_{1}-t_{2}\right)
$$

Finally, substituting (31) and (25) into (G3) implies (39).

\section{In the time-frequency domain}

Finally, from (F1) and (17), since

$$
\int_{u \in \mathbb{R}} \int_{\boldsymbol{x} \in \mathbb{R}^{3}} \frac{\left|g\left(t+\frac{u}{2}-T-\frac{\left\|\boldsymbol{x}-\boldsymbol{x}_{i}\right\|_{2}}{c}\right) g\left(t-\frac{u}{2}-T-\frac{\left\|\boldsymbol{x}-\boldsymbol{x}_{j}\right\|_{2}}{c}\right)\right|}{\left\|\boldsymbol{x}-\boldsymbol{x}_{i}\right\|_{2}\left\|\boldsymbol{x}-\boldsymbol{x}_{j}\right\|_{2}} d \boldsymbol{x} d u<+\infty
$$

Fubini's theorem shows that we can permute the mathematical expectation with the integrals over $\boldsymbol{x}$ in (18) and over $u$ in (11) (with $\psi_{1}=h_{i}$ and $\psi_{2}=h_{j}$ ), leading to:

$$
\mathcal{W}_{h_{i}, h_{j}}(t, f)=\lambda e^{-2 \alpha(t-T)} \int_{\mathbb{R}} J_{\xi_{1}, \xi_{2}} e^{-2 \imath \pi f u} d u
$$

where $\xi_{1}(r)=g\left(t+\frac{u}{2}-T-\frac{r}{c}\right), \xi_{2}(r)=g\left(t-\frac{u}{2}-T-\frac{r}{c}\right)$, and $J_{\xi_{1}, \xi_{2}}$ was defined in (E1).

Then substituting (E3) into (G4) shows that

$$
\mathcal{W}_{h_{i}, h_{j}}(t, f)=\frac{4 \pi \lambda e^{-2 \alpha(t-T)}}{2 D} \int_{\rho=\frac{D}{2}}^{+\infty} \int_{v=-D}^{D} I\left(t-T-\frac{\rho}{c}, f, v\right) d \rho d v
$$

with

$$
I(t, f, v)=\int_{u \in \mathbb{R}} g\left(t+\frac{u}{2}-\frac{v}{2 c}\right) g\left(t-\frac{u}{2}+\frac{v}{2 c}\right) e^{-2 \imath \pi f u} d u=e^{-2 \imath \pi f \frac{v}{c}} \mathcal{W}_{g, g}(t, f)
$$

where $\mathcal{W}_{g, g}(t, f)$ was expressed in (D3), and we have used the change of variable $u^{\prime}=u-\frac{v}{c}$. 
In order to conclude, we will use the two properties of the Wigner distribution that we already used in Appendix D $2 \mathrm{c}$. Firstly, the time support of $\mathcal{W}_{g, g}$ is the same as that of $g$. Secondly, $\int_{t \in \mathbb{R}} \mathcal{W}_{g, g}(t, f) d t=\left|\mathcal{F}_{g}(f)\right|^{2}$. Substituting (G6) and (26) into (G5) finally implies (40).

\section{Asymptotic normality}

Since $\boldsymbol{b}(t)$ converges in law to a stationary Gaussian process as shown in Lemma 2, equation (17) shows that $\boldsymbol{h}(t)$ converges in law to a Gaussian process.

${ }^{1}$ The Fourier transform is defined in equation (9).

${ }^{2}$ The correlation of two complex random variables is defined in equation (7).

${ }^{3}$ Note that in this case, $\mathcal{F}_{h_{i}}(f)$ is no longer a stationary process.

${ }^{4}$ The Wigner distribution is defined in equation (11).

${ }^{5} \mathrm{~A}$ straightforward generalization of this model will also permit us to prove (5), that will be presented in a future paper .

${ }^{6}$ Fig. 3 illustrates the source image principle in 2D-space for convenience, but of course our model will be defined in the 3D-space.

${ }^{7}$ For the sake of simplicity, we will focus on the case of two microphones; the generalization to an arbitrary number of microphones is straightforward.

${ }^{8}$ More precisely, (15) is required to prove that the random processes $b_{i}(t)$ and $h_{i}(t)$ are centered after a given time ( $c f$. Appendix C 1 a), and (16) enforces the nonnegativity of $\mathcal{L}_{g}(\alpha+2 \imath \pi f)$ in (20), which makes 
${ }^{11}$ An example of function $\psi(t)$ satisfying the conditions in Lemma 3 is

$$
\psi(t)=\left(\left(\alpha^{2}+\frac{4 \pi^{2}}{T^{2}}\right) \cos \left(\frac{2 \pi t}{T}\right)-\alpha^{2}\right) \mathbf{1}_{[0, T]}(t)
$$

${ }_{527}{ }^{12} \mathrm{~A}$ simple function is defined as a linear combination of indicator functions of measurable sets.

${ }_{528}{ }^{13}$ The proof is the same and it is omitted here for the sake of conciseness.

529 Proposition 2 that simplifies to Schroeder's equation (2) when $T \rightarrow 0$.

${ }^{9} \mathrm{~A}$ WSS process is a random process with finite second order moments, whose mean and covariances are invariant with respect to any translation of time.

Wigner distributions are estimated: the STFT produces estimated time-frequency distributions $\widehat{\mathcal{W}}_{h_{i}, h_{i}}(t, f)$ and $\widehat{\mathcal{W}}_{h_{i}, h_{j}}(t, f)$ that are smoothed both in time and frequency. Therefore the estimate (42) is the ratio between two spectra that are both smoothed in frequency, which explains the deviations between the two curves in Fig. 5-(a).

Allen, J. B., and Berkley, D. A. (1979). "Image method for efficiently simulating small-room acoustics," Journal of the Acoustical Society of America 65(4), 943-950.

Balian, R., and Bloch, C. (1970). "Distribution of eigenfrequencies for the wave equation in a finite domain: I. three-dimensional problem with smooth boundary surface," Annals of Physics 60(2), 401-447.

Baskind, A. (2003). "Modèles et méthodes de description spatiale de scènes sonores : application aux enregistrements binauraux (Models and methods of spatial description of sound

the filter $A(f)$ linear-phase, and which is required to get an expression $(30)$ of $\operatorname{corr}\left[\mathcal{F}_{h_{i}}\left(f_{1}\right), \mathcal{F}_{h_{i}}\left(f_{2}\right]\right)$ in 
JASA/Common mathematical framework for stochastic reverberation models

scenes: application to binaural recordings)," Ph.D. thesis, Université Pierre et Marie Curie (UPMC), Paris, France.

Chiu, S. N., Stoyan, D., Kendall, W. S., and Mecke, J. (2013). Stochastic Geometry and Its Applications, Chap. 2 "Point processes I - The Poisson point process", 3rd edition ed. (Wiley, Hoboken, NJ, USA).

Cohen, L. (1989). "Time-frequency distributions-a review," Proc. IEEE 77(7), 941-981. Cook, R. K., Waterhouse, R. V., Berendt, R. D., Edelman, S., and Thompson Jr., M. C. (1955). "Measurement of correlation coefficients in reverberant sound fields," The Journal of the Acoustical Society of America 27(6), 1072-1077.

Cremer, L., Müller, H. A., and Schultz, T. J. (1982). Principles and applications of room acoustics, 1, Chap. (ii) "Statistical Room Acoustics" (Applied Science Publishers, London, $\mathrm{UK})$.

Elko, G. W. (2001). "Spatial coherence functions for differential microphones in isotropic noise fields," Microphone Arrays 61-85.

Jacobsen, F., and Roisin, T. (2000). "The coherence of reverberant sound fields," The Journal of the Acoustical Society of America 108(1), 204-210.

Jot, J.-M., Cerveau, L., and Warusfel, O. (1997). "Analysis and synthesis of room reverberation based on a statistical time-frequency model," in AES Convention 103, Audio Engineering Society, New, NY, USA, pp. 4629-4658.

Joyce, W. B. (1975). "Sabine's reverberation time and ergodic auditoriums," The Journal of the Acoustical Society of America 58(3), 643-655. 
JASA/Common mathematical framework for stochastic reverberation models

Kuttruff, H. (2014). Room Acoustics, Fifth Edition (CRC Press, Boca Raton, FL, USA), pp. 1-374.

Maa, D.-Y. (1939). "Distribution of eigentones in a rectangular chamber at low frequency range," The Journal of the Acoustical Society of America 10(3), 235-238.

Moorer, J. A. (1979). "About this reverberation business," Computer Music Journal 3(2), $13-28$.

Polack, J. D. (1988). "La transmission de l'énergie sonore dans les salles (The transmission of sound energy in rooms)," Ph.D. thesis, Université du Maine, Le Mans, France.

Polack, J.-D. (1992). "Modifying chambers to play billiards: the foundations of reverberation theory," Acta Acustica united with Acustica 76(6), 256-272(17).

Polack, J.-D. (1993). "Playing billiards in the concert hall: The mathematical foundations of geometrical room acoustics," Applied Acoustics 38(2), 235-244.

Polack, J.-D. (2007). "The relationship between eigenfrequency and image source distributions in rectangular rooms," Acta Acustica united with Acustica 93, 1000-1011.

Schroeder, M. R. (1962). "Frequency-correlation functions of frequency responses in rooms," The Journal of the Acoustical Society of America 34(12), 1819-1823.

Schroeder, M. R. (1987). "Statistical parameters of the frequency response curves of large rooms," The Journal of the Audio Engineering Society 35(5), 299-306.

Schroeder, M. R., and Kuttruff, K. H. (1962). "On frequency response curves in rooms. Comparison of experimental, theoretical, and Monte Carlo results for the average frequency spacing between maxima," The Journal of the Acoustical Society of America 34(1), 76-80. 
JASA/Common mathematical framework for stochastic reverberation models

579 Schultz, T. (1971). "Diffusion in reverberation rooms," Journal of Sound and Vibration $580 \quad \mathbf{1 6}(1), 17-28$.

${ }_{581}$ Vincent, E., and Campbell, D. R. (2008). "Roomsimove" GNU Public License, http:// homepages.loria.fr/evincent/software/Roomsimove_1.4.zip. 